\title{
THE MAGNETIC FIELD INSIDE THE SUN
}

\author{
W. Dziembowski \\ N. Copernicus Center, Polish Academy of Sciences, \\ Warsaw, Poland \\ Philip R. Goode \\ Department of Physics, New Jersey Institute of Technology \\ Newark, New Jersey, USA
}

\begin{abstract}
Duvall, Harvey and Pomerantz (1986) reported the existence of a 'structural asymmetry' inside the Sun. We show that this asymmetry is not a consequence of the Sun's rotation. We attribute the asymmetry, rather, to a toroidal magnetic field inside the Sun. Consistency requires a field of about one megagauss located in the lower part of the convection zone. Accounting for such a field would wreak havoc on our understanding of the solar dynamo and convection.
\end{abstract}

Duvall, Harvey and Pomerantz (1986, hereafter denoted by DHP) determined that there is no apparent variation in rotation from the surface through the convection zone of the Sun. This result is consistent with that of Duvall et al (1984) which applies for the equatorial plane of the Sun. The DHP result followed from their observations and study of oscillations having degree between 20 and 98 . In fact, their study implied that the surface differential rotation rate persists even below the convection zone.

DHP presented their frequency splitting using

$$
\nu(n, \ell, m)-\bar{\nu}(n, \ell, 0)=\sqrt{\ell(\ell+1)} \sum_{\mathrm{i}=0}^{\mathrm{N}} a_{\mathrm{i}} P_{\mathrm{i}}[-m / \sqrt{\ell(\ell+1)}],
$$

where $\bar{\nu}$ is the mean frequency for a particular $\ell$ and the $P_{\mathrm{i}}$ are Legendre polynomials. Table 1 shows their result. The advantage of a Legendre expansion over a polynomial in $m$ is that the former yields increasingly orthogonal terms as $\ell$ increases. The DHP rotation law follows from the odd coefficients in the table because the rotation rate can be written as

$$
\Omega(r, \mu)=\Omega_{0}(r)+\Omega_{1}(r) \mu^{2}+\Omega_{2}(r) \mu^{4}+\cdots, \mu=\cos \theta .
$$

For the DHP data, the series cuts off with $\Omega_{2} ; \Omega_{0}, \Omega_{1}$ and $\Omega_{2}$ are independent of radius. The even coefficients in the table reflect the 'structural asymmetry'. We show here that this asymmetry is not due to the second order effects of rotation, to which the largest two contributions arise from the Coriolis force and the centrifugal effects of rotation on the equilibrium shape of the Sun. In the notation of DHP, the magnitude of the second order Coriolis term is approximated by 
TABLE 1 Average rotation coefficients and standard deviations (nHz)

\begin{tabular}{|c|c|c|c|c|c|}
\hline$\ell$ range & $a_{1}$ & $a_{2}$ & $a_{3}$ & $a_{4}$ & $a_{5}$ \\
\hline $20-29$ & $446.1 \pm 2.7$ & $18.2 \pm 3.4$ & $20.8 \pm 4.1$ & $0.4 \pm 4.7$ & $-5.2 \pm 5.3$ \\
\hline 30-39 & 444.51 .5 & 4.52 .0 & 20.12 .4 & $\begin{array}{lll}8.7 & 2.7\end{array}$ & -6.23 .0 \\
\hline $40-49$ & $445.4 \quad 1.1$ & 8.21 .5 & 20.21 .8 & 3.42 .0 & -1.82 .2 \\
\hline 50-59 & 442.31 .0 & 8.21 .3 & 23.01 .5 & $2.8 \quad 1.7$ & $\begin{array}{lll}-7.4 & 1.9\end{array}$ \\
\hline $60-69$ & 444.20 .9 & $\begin{array}{lll}8.6 & 1.2\end{array}$ & $20.8 \quad 1.4$ & 4.01 .6 & $\begin{array}{lll}-3.5 & 1.8\end{array}$ \\
\hline $70-79$ & $\begin{array}{lll}444.4 & 0.8\end{array}$ & $\begin{array}{lll}4.4 & 1.0\end{array}$ & 21.41 .2 & $\begin{array}{lll}3.5 & 1.4\end{array}$ & -3.91 .5 \\
\hline $80-89$ & $446.0 \quad 0.9$ & 4.01 .1 & 20.01 .4 & $3.0 \quad 1.5$ & $\begin{array}{lll}-3.0 & 1.7\end{array}$ \\
\hline $90-98$ & 444.01 .0 & $\begin{array}{lll}6.4 & 1.3\end{array}$ & 22.91 .5 & $\begin{array}{ll}-2.9 & 1.7\end{array}$ & $-1.7 \quad 1.9$ \\
\hline
\end{tabular}

$$
a_{2, \mathrm{c}} \sim \frac{\ell \Omega_{1}^{2}}{2 \omega_{0}} \sim 0.1 \mathrm{nHz} .
$$

The term $\Omega_{1}$ is the coefficient of the $\mu^{2}$-term in the rotation law because the coefficient of the larger $\mu^{0}$-term would be multiplied by the Ledoux constant which is quite small. The centrifugal stretching terms is approximated by

$$
a_{2, \mathrm{~d}} \sim \frac{3\left\langle\varepsilon>\omega_{0}\right.}{\ell} \sim 0.5 \mathrm{nHz},
$$

where $\langle\varepsilon\rangle$ is the magnitude of the mean centrifugal distortion of the Sun. Our detailed calculation of the $a_{2, \mathrm{~d}}$-term reveals that it varies between -1.0 $\mathrm{nHz}$ (at $\ell=25$ ) and $-0.3 \mathrm{nHz}$ (at $\ell=95$ ). This is far to small to account for the data. The $a_{4}$-term from rotation is considerably smaller still.

We assume that a toroidal magnetic field is responsible for the asymmetry. This assumption is consistent with the latitudinal geometry of sunspots and the expectation that differential rotation would shear a poloidal field into a toroidal field with the latter growing with time (Mestel and Moss, 1977). For instance, a minuscule relic poloidal field could be sheared into a large toroidal field. We define the field, $B_{\mathrm{T}}$, in general terms by

$$
\frac{B_{T}^{2}}{4 \pi p}=\sin ^{2} \theta \sum_{\mathrm{k}=0} \beta_{\mathrm{k}}(r) \mu^{2 \mathrm{k}} \text {. }
$$

The $\beta_{\mathrm{k}}(r)$-term and the pressure, $p$, define the radial dependence of the field. The field changes the frequencies directly through its perturbation of the Lorentz force and indirectly through its effects on the equilibrium model. In particular, the perturbation of the gravitational potential effects the whole star resulting in a change of frequency even for modes which do not interact with the field. Including all the aforementioned effects, we write kernels in the form

$$
a_{\mathrm{j}}=\sum_{\mathrm{k}} \int \beta_{\mathrm{k}}(r) K_{\mathrm{jk}} \frac{d r}{c}, j=2,4, \cdots 2 k+2,
$$

where $c$ is the local speed of sound and the $a$ 's are as defined by DHP. These expressions are completely general for the toroidal field and the $K_{j \mathrm{k}}$ may be calculated for individual modes, or as in the present case, for a 


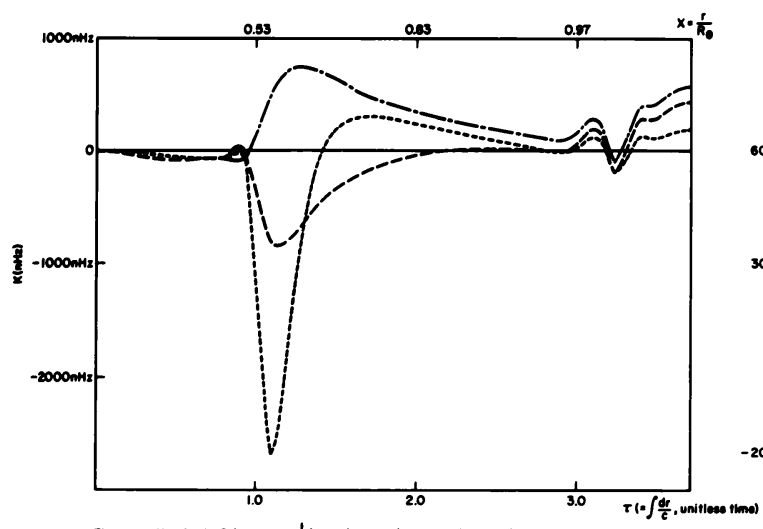

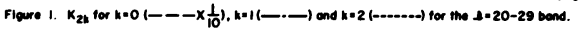
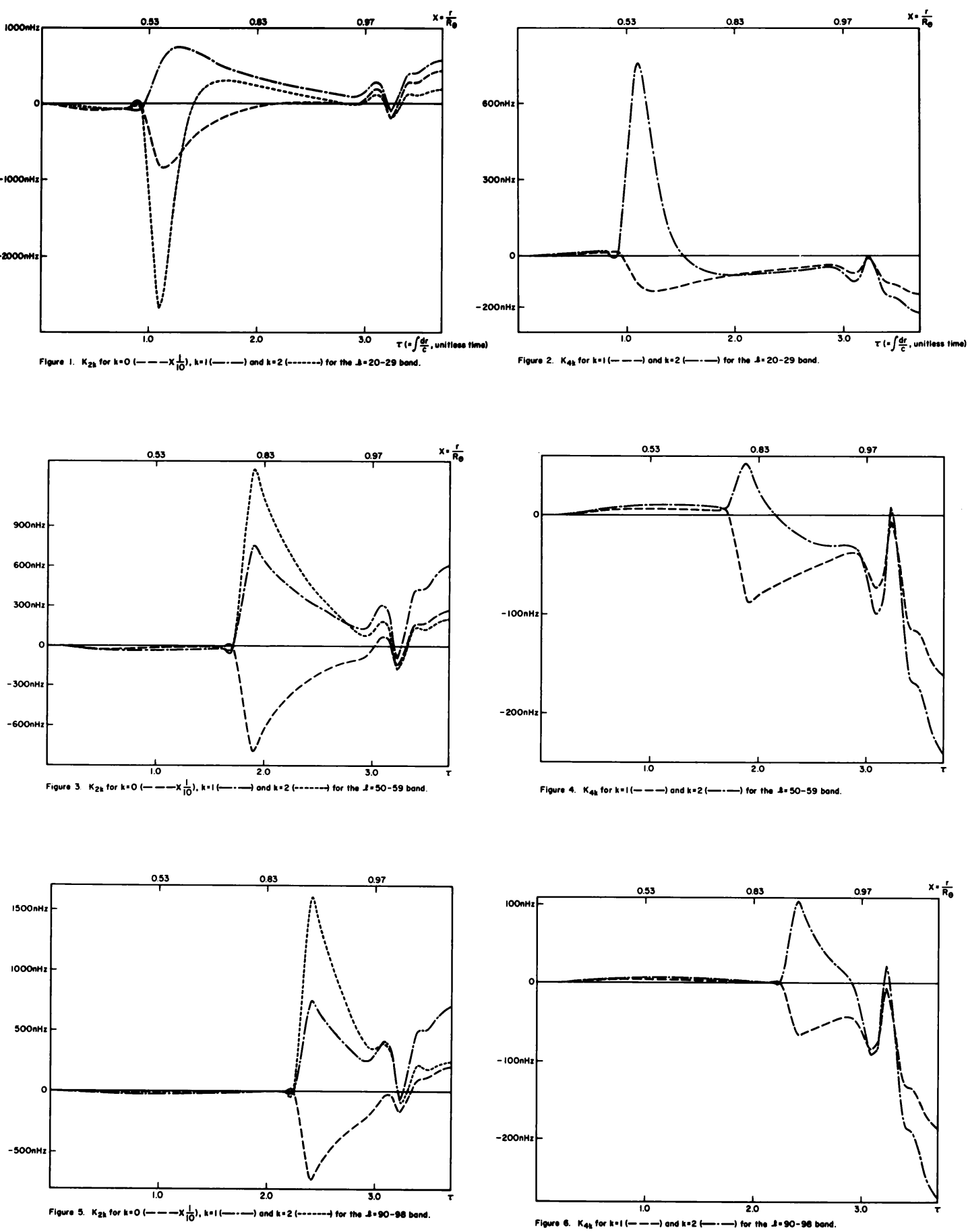
group of modes. The $a_{\mathrm{j}}$ - and $K_{\mathrm{jk}}$ - terms are measured in $\mathrm{nHz}$ and the remaining terms in the integrand are unitless with time being measured in units of $1 / \sqrt{4 \pi G\langle p\rangle}$ where $\langle p\rangle$ is the mean solar density.

We have calculated the $K_{\mathrm{jk}}$ in a rough approximation, assuming that the radial derivative of the oscillation energy could be replaced by its envelope value, i.e. $1 / c$ for $r\rangle r_{t}$ and 0 for $r\left\langle r_{t}\right.$. The inner turning point, $r_{t}$, has been determined from the condition $2 \pi \nu=\sqrt{\ell(\ell+1)} c\left(r_{t}\right) / r_{t}$, where $c\left(r_{t}\right)$ is the speed of sound at the turning point and $\nu$ is the frequency of the mode. We chose $3 \mathrm{mHz}$ for this frequency and the average $\ell$-value for each $\ell$-band of DHP. We calculated kernels in this way for $k=1,2$ and 3 for $j=2,4$ and 6 . These kernels are plotted in Figures 1 to 6 for the $\ell=20-29,50-59$ and 90-98 bands for $j=2$ and 4. The kernels for $j=6$ are much smaller and are not shown. The values for the kernels shown in the figures are appreciably smaller than expected from a simple order-of-magnitude estimate to account for $a_{2}$ and $a_{4}$. It can be seen that $\beta^{\prime} s$ of the order of $10^{-2}-10^{-3}$ would be required if the field were located in the convective envelope. Considerably larger $\beta^{\prime} s$ would be required if the field were located in the core. The kernels tend to peak near the inner turning point. This fact and the larger value of $a_{2}$ for the $\ell=20-29$ band require a field on the order of one megagauss in the lower part of the convection zone. Such a field would play a significant role in the motion in that part of the convection zone. This can be seen by estimating the square of the ratio of the Alfven velocity to the Brunt-Văisălă frequency times radius, that is,

$$
\frac{B_{\mathrm{T}}^{2}}{4 \pi \rho|N|^{2} r^{2}} \sim 10^{2}
$$

Arbitrarily assuming the field were located just beneath the surface implies it would have to be about a kilogauss - such a field would be difficult to imagine as being stabilized over the 11 year solar cycle. The structure in the kernels near the model surface arises from derivatives of $\Gamma$ which are the results of eliminating radial derivatives of the field.

The next stage of this work is underway and is a precise calculation of the toroidal field's role in frequency splitting. We do not expect, however, that the results will differ much from those presented here. Equivalent calculations will be done for a poloidal field.

We thank Drs Werner Dăppen and Don Kurtz for critical reading of the paper. We thank the Research Office at the New Jersey Institute of Technology for financial support which facilitated this work. Helene Nielsen is thanked for retyping the paper at very short notice.

\section{References:}

Duvall, T.L. Jr., Dziembowski, W.A., Goode, P.R., Gough, D.O., Harvey, J.W. and Leibacher, J.W., Nature, 310, 22 (1984).

Duvall, T.L. Jr., Harvey, J.W. and Pomerantz, M.A., Nature, 321, 500 (1986).

Mestel, L. and Moss, D.L., Mon. Not. R. Astr. Soc. 178, 27 (1977). 